\title{
Magnetic bead micromixer: Influence of magnetic element geometry and field amplitude
}

\author{
Lund-Olesen, Torsten; Buus, Bjarke B.; Howalt, Jakob; Hansen, Mikkel Fougt
}

Published in:

Journal of Applied Physics

Link to article, DOI:

$10.1063 / 1.2829401$

Publication date:

2008

Document Version

Publisher's PDF, also known as Version of record

Link back to DTU Orbit

Citation (APA):

Lund-Olesen, T., Buus, B. B., Howalt, J., \& Hansen, M. F. (2008). Magnetic bead micromixer: Influence of magnetic element geometry and field amplitude. Journal of Applied Physics, 103(7), $07 \mathrm{E} 902$.

https://doi.org/10.1063/1.2829401

\section{General rights}

Copyright and moral rights for the publications made accessible in the public portal are retained by the authors and/or other copyright owners and it is a condition of accessing publications that users recognise and abide by the legal requirements associated with these rights.

- Users may download and print one copy of any publication from the public portal for the purpose of private study or research.

- You may not further distribute the material or use it for any profit-making activity or commercial gain

- You may freely distribute the URL identifying the publication in the public portal

If you believe that this document breaches copyright please contact us providing details, and we will remove access to the work immediately and investigate your claim. 


\title{
Magnetic bead micromixer: Influence of magnetic element geometry and field amplitude
}

\author{
Torsten Lund-Olesen, ${ }^{\text {a) }}$ Bjarke B. Buus, Jakob G. Howalt, and Mikkel F. Hansen ${ }^{\text {b) }}$ \\ MIC-Department of Micro and Nanotechnology, Technical University of Denmark, Building 345 east, \\ DK-2800 Kongens Lyngby, Denmark
}

(Presented on 9 November 2007; received 6 September 2007; accepted 4 October 2007; published online 23 January 2008)

\begin{abstract}
A scheme for the silicon microfabrication of lab-on-a-chip systems with mixing based on dynamic plugs of magnetic beads is presented. The systems consist of a microfluidic channel integrated with a number of soft magnetic elements by the sides of the channel. The elements are magnetized by a homogeneous external ac magnetic field. The systems are scalable with respect to the number of magnetic bead plugs and number of parallel channels, and thus they have high potential for use in biological separation using functionalized magnetic beads. The mixing efficiency is characterized for two different geometries of the soft magnetic structures and found to be highly sensitive to the geometry and position of the structures. (C) 2008 American Institute of Physics.

[DOI: $10.1063 / 1.2829401]$
\end{abstract}

The use of magnetic beads for magnetic bioseparation in lab-on-a-chip systems is gaining interest because the beads can be manipulated by external magnetic forces independent of the biology and chemistry taking place in the system and because they can provide a large surface area for biological separation. Rida and Gijs have proposed and demonstrated a micromixer based on a dynamic plug of magnetic beads agitated by an ac magnetic field in a microfluidic channel with a fluid flow. ${ }^{1}$ The implementation of mixers in microfluidic systems with dimensions on the order of $100 \mu \mathrm{m}$ is important as the liquid flow is usually laminar and typical diffusion times are on the order of minutes to hours. The movement of the beads in the dynamic bead plug creates transverse movement of liquid, which causes mixing of the liquid. In addition to mixing, it has been proposed to use this principle in labon-a-chip system for bioseparation using functionalized magnetic beads. The design by Rida and Gijs presented in Ref. 1 was made using powder blasting of polymethylmethacrylate and laser cutting of metal. The magnetic elements were made to be in direct contact with a small electromagnet to form a closed magnetic circuit. They studied the mixing efficiency as function of magnetic field amplitude and frequency and concluded that mixing efficiency was highest for low magnetic field amplitude and low frequency approaching the limit where the beads are released. ${ }^{1}$

We present and characterize two dynamic bead plug mixers fabricated using silicon micromachining. The fabrication is based on deep reactive ion etching (DRIE) in silicon and electroplating of soft magnetic elements. This facilitates batch fabrication of microsystems and makes the mixer structures easy to integrate with other microfluidic structures. The presented mixer also has the ability to contain many bead plugs and can easily be expanded to have several parallel microfluidic separation channels. The design uses an external magnetic field instead of direct contact between

\footnotetext{
${ }^{a)}$ Electronic mail: tlo@mic.dtu.dk.

${ }^{b)}$ Electronic mail: mfh@mic.dtu.dk
}

electromagnet and magnetic elements. We present the fabrication process and characterize the performance of two systems with different geometries of the soft magnetic elements as a function of the applied field amplitude. We show that the element geometry has a strong influence on the mixing efficiency as well as the ability to retain magnetic bead plugs at low magnetic field amplitudes.

The mixer principle is described in detail in Ref. 1. A microfluidic channel has soft magnetic elements on each side that retain a plug of magnetic beads when they are magnetized by an external magnetic field. When the magnetic field amplitude is low, the beads are released and move along the fluid flow in the channel. Upon application of an ac magnetic field, the beads move around in circular paths and provide the mixing of the fluid in the channel. Our chip is placed in a homogenous external ac magnetic field provided by an external electromagnet. This has the advantage that the number of channels and elements on a chip can easily be scaled and the disadvantage compared to the design of Rida and Gijs $^{1}$ that the field amplitude for a given current is lower due to the higher reluctance of our magnetic circuit from the extra gaps. Figure 1 shows micrographs of part of the two chips used in the presented experiments. The chips are made in silicon with a glass lid and have a size of $1 \times 3 \mathrm{~cm}^{2}$. The microfluidic channel has a cross section of $h \times w=200$ $\times 200 \mu \mathrm{m}^{2}$ and the magnetic elements are placed with a periodicity of $1 \mathrm{~mm}$ along the sides of the channel. They are $\approx 4.8 \mathrm{~mm}$ long, $\approx 200 \mu \mathrm{m}$ high, and $600 \mu \mathrm{m}$ wide ending in different geometries toward the channel.

Figure 2 schematically illustrates the fabrication procedure, which is based on those reported in Refs. 2 and 3. Further development has been made to obtain soft magnetic elements over the entire height of the fluid channel. The labels below refer to the figure. The starting wafer is a $350 \mu \mathrm{m}$ thick double polished silicon wafer. (a) First, a $2 \mu \mathrm{m}$ wet thermal oxide is grown and patterned using photolithography and buffered HF (BHF) etch. The resulting oxide mask has openings where the fluid channel and the mag- 

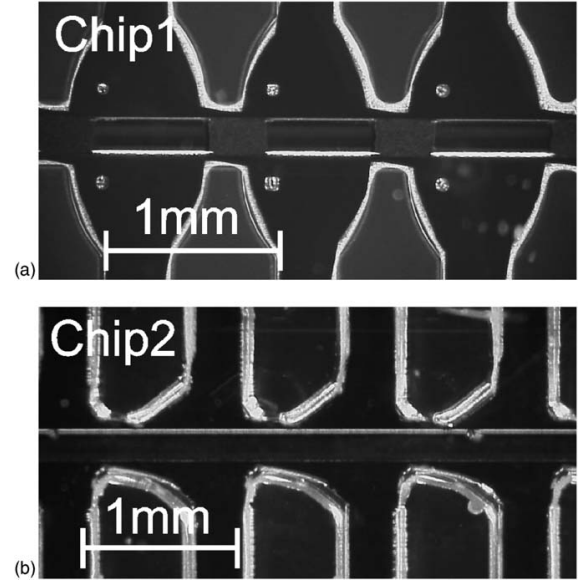

FIG. 1. Micrographs of part of the two microsystems used in the presented experiments. In (a) plugs of beads can be seen in the channel. No beads are present in (b).

netic elements are situated. Then, a second mask of $6.5 \mu \mathrm{m}$ AZ4562 photoresist (Hoechst, NJ) is patterned. This mask has openings only where the magnetic elements are situated. (b) Subsequently, two DRIE steps are performed: first, $100 \mu \mathrm{m}$ of silicon is etched using the resist mask and then, after removal of the resist mask in acetone, $200 \mu \mathrm{m}$ of silicon is etched using the oxide mask. (c) Subsequently, the oxide mask is removed in BHF, an RCA clean is performed, a $170 \mathrm{~nm}$ dry thermal oxide is grown, and a borofloat glass wafer is anodically bonded to the silicon wafer sealing the fluid channel and the trenches for the elements. (d) The oxide is removed in BHF. As the structures on the front side are sealed by the glass wafer only the oxide on the back side is removed. (e) KOH etching is performed until all the structures for magnetic elements are open to the back side of the wafer. This fabrication step has caused problems. The DRIE etches are not uniform all over the wafer which means that the silicon will be etched through at some places on the wafer before others. Once a hole has been etched, $\mathrm{KOH}$ enters the structures. The oxide on the sidewalls protects the
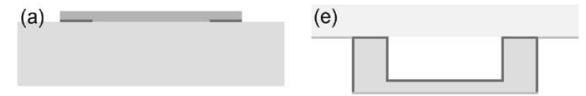

(b)
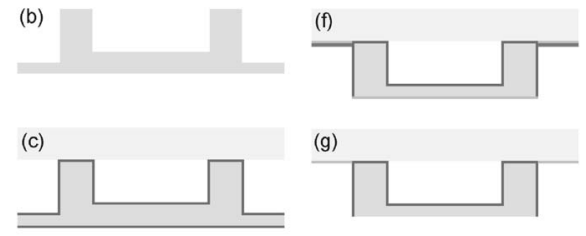

(g)

(d)
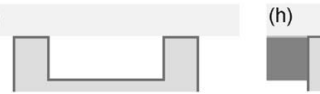

FIG. 2. Fabrication scheme (cross-section view). (a) The starting wafer with two masks (an oxide mask and on top of that a photoresist mask). (b) Two steps of DRIE using the two masks. (c) RCA-clean, $170 \mathrm{~nm}$ thermal oxide growth and anodic bonding to a glass wafer. (d) BHF etch of the back side oxide followed by $\mathrm{KOH}$ etch to open the magnetic element structures. (e) Seed layer deposition $(5 / 200 \mathrm{~nm} \mathrm{Ti} / \mathrm{Au}$, e-beam evaporation). (f) Electroplating of a $\mathrm{Cu}$ protection layer. (g) Etching of Au in Entreat followed by removal of the $\mathrm{Cu}$ protection layer in nitric acid. (h) Electroplating of Permalloy followed by dicing of the wafer into individual chips.

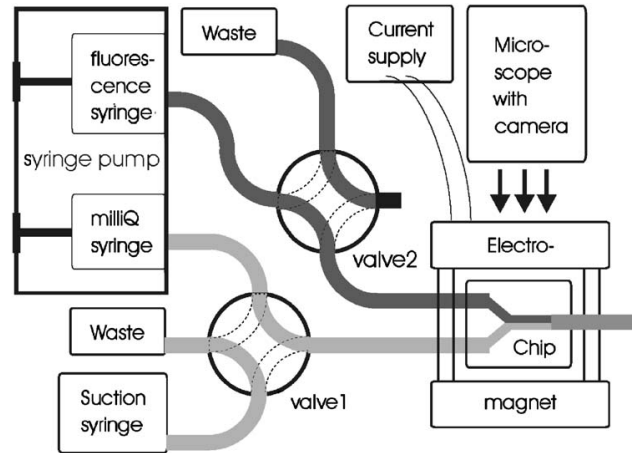

FIG. 3. Schematic of the setup.

silicon from etching for a while but will eventually be etched through. For the present systems some etching of the silicon occurred that slightly changed the geometry and the distance between magnetic elements and channel. A thicker oxide or more uniform etching depth in the DRIE steps can remove this problem. (f) A $5 / 200 \mathrm{~nm} \mathrm{Ti} / \mathrm{Au}$ seed layer is e-beam evaporated on the back side with intentionally low step coverage. (g) Electroplating of $\approx 3 \mu \mathrm{m} \mathrm{Cu}$. Due to the poor step coverage of the seed layer, $\mathrm{Cu}$ is only electroplated at the bottom of the structures. (h) Removal of Au not protected by $\mathrm{Cu}$ in Entreat 100 (Engelhard, NJ) followed by removal of the $\mathrm{Cu}$ in nitric acid. This leaves a $\mathrm{Ti} / \mathrm{Au}$ seed layer only in the trenches for the magnetic elements. (i) Electroplating of Permalloy to the same height as that of the fluid channel. The last steps are dicing of the wafer into individual chips and drilling of fluidic access holes using a diamond drill.

The magnetic beads used in the experiments are MagNA pure LC magnetic glass particles (Roche Diagnostics, Switzerland). The beads are $1-10 \mu \mathrm{m}$ in diameter with a mean diameter of $3 \mu \mathrm{m}$, have a saturation magnetization of $160 \mathrm{kA} / \mathrm{m}$, and a remanent magnetization of $32 \mathrm{kA} / \mathrm{m}^{1}$. The bead solution used in the experiments was the original bead solution diluted three times with milliQ water.

Figure 3 shows a schematic of the setup. All images are acquired using a Leica MZ-FLIII stereo microscope equipped with a Sony DFW-X810 charge coupled device camera. An experiment is performed as follows: (1) The sinusoidal external ac magnetic field is turned on with a frequency of $10 \mathrm{~Hz}$ and a rms value of $\approx 23 \mathrm{mT}$. (2) Valve 1 is turned so the suction syringe is connected to the chip and valve 2 so the other inlet on the chip is blocked. (3) $35 \mu \mathrm{l}$ bead solution is pulled into the chip from the outlet using the suction syringe (most of this volume is used to fill the tube connected to the outlet). (4) The syringe pump is started at a flow rate of $10 \mu \mathrm{l} / \mathrm{min}$. Syringe 1 pushes milliQ water into the system and the fluorescent solution from syringe 2 is pushed to waste. (5) After beads no longer enter the system and the amount of beads in each plug has stabilized, the flow rates from the two syringes are changed to $4 \mu \mathrm{l} / \mathrm{min}$ and valve 2 is switched so both liquids are pushed into the chip giving a total flow rate of $8 \mu \mathrm{l} / \mathrm{min}$ of which half is fluorescent. (6) After the fluorescence flow has stabilized and the fluorescence at the inlets is constant in time, pictures are taken at the inlet for determining the inlet fluorescence distribution $\left(I_{b m, n}\right)$. A reference picture is taken with normal 


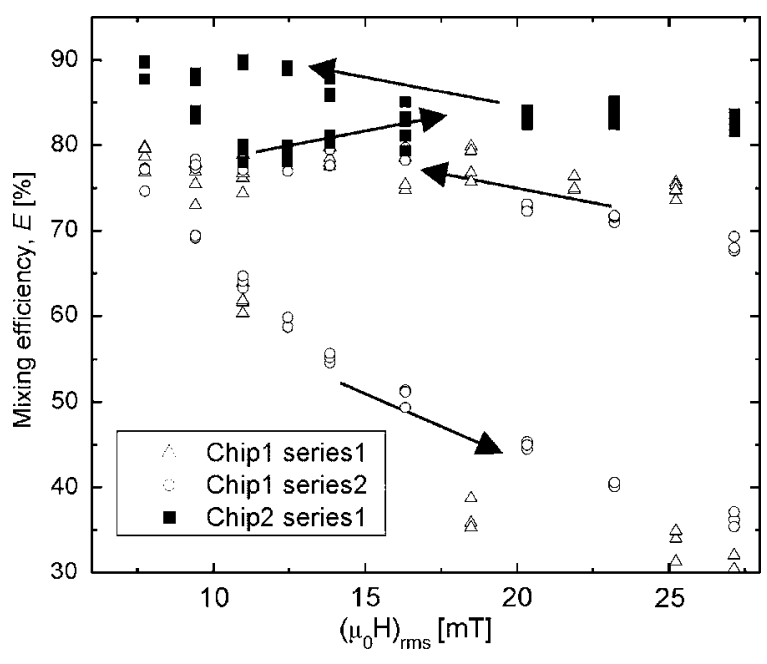

FIG. 4. Measured mixing efficiencies as a function of rms value of the external sine ac magnetic field. The arrows indicate the sequence of measurements.

light for identifying the channel sidewalls. (7) The chip is moved and a reference picture is taken between elements 9 and 10 for identifying the channel side walls. (8) A series of pictures is taken to determine fluorescence distributions $\left(I_{a m, n}\right)$ for different settings. Three pictures are taken for each setting of parameters. In the experiments the rms value of the magnetic field is changed from high $(25-27 \mathrm{mT})$ down to low $(\approx 7.6 \mathrm{mT})$ and back up again. (9) Valve 1 is switched. This means only that fluorescent liquid enters the channel and after stabilization a picture is taken and the obtained fluorescence distribution $\left(I_{\infty, n}\right)$ is used as a reference for complete mixing.

After a series of experiments, each image was analyzed automatically using a MATLAB script to get normalized fluorescence distributions and mixing efficiencies calculated using Eq. (1).

$$
E=\left(1-\frac{\sum_{n}\left|I_{a m, n}-I_{\infty, n}\right|}{\sum_{n}\left|I_{b m, n}-I_{\infty, n}\right|}\right) .
$$

To remove optical effects at the sides of the channel $6 \%$ on each side of the channel is removed from this calculation.

Figure 4 shows graphs of mixing efficiencies as a function of first decreasing and then increasing amplitude of the magnetic field.

On chip 1, two series of measurements were performed several days apart and after remounting the chip. It is seen that the results are reproducible within about $5 \%$. Starting at high fields, the mixing efficiency is around $70 \%$ and then increases to about $80 \%$ when the rms value of the magnetic field is lowered to $11-14 \mathrm{mT}$. When it is lowered further to about $8 \mathrm{mT}$ the efficiency changes only little, but it is observed that magnetic beads are lost from the plugs. Upon an increase of the field, the mixing efficiency is observed to steadily decrease reaching about $35 \%$ at the maximum rms value of $27 \mathrm{mT}$. This decrease is due to loss of beads from the plugs, which then become compact and less mobile at higher fields.
Chip 2, in contrast, shows a different behavior. Starting at high fields the mixing efficiency is slightly below $85 \%$ and increases to about $90 \%$ at low fields where again some beads are seen to be lost. When the rms value of the field is subsequently increased, the mixing efficiency first decreases to slightly below $80 \%$ around $11-12 \mathrm{mT}$ and then increases to slightly below $85 \%$ and retraces the initial curve at higher fields. Thus, for chip 2 the mixing efficiency displays a very different dependency on magnetic field amplitude and amount of beads in a plug.

The reason for the difference between chips 1 and 2 is the element geometry and placement. Chip 1 has symmetric elements with $90^{\circ}$ corners that result in very strong but also localized magnetic field gradients that only provide strong retaining forces on the beads on a small portion of the length of the channel making the bead plug small and dense. In chip 2 the asymmetric element geometry results in retaining forces acting against the fluid flow direction over a significant portion of the length of the channel. The bead plugs in this system are larger and less dense. The element shape, however, is not the entire explanation. Due to small differences in the way the two chips were fabricated, the elements in chip 2 are placed closer to the channel than chip 1 and other experiments suggest that this factor has the largest influence (data not shown).

In the experiments, it was also noticed in both systems that the bead plug after the last element was about twice the size of those in the middle of the array of magnetic elements. This shows that larger bead plugs can be obtained if the spacing between the elements is larger and thus that a higher mixing efficiency may be obtained with a lower density of the elements. This is in agreement with the results of Rida and Gijs, ${ }^{1}$ who found high mixing efficiencies for just a single plug of beads. However, it is not clear what the optimum density of the elements is for maximum capture efficiency and binding capacity when used for biomagnetic separation.

We presented a fabrication sequence that enables fabrication in silicon of micromixers using dynamic plugs of beads. The fabrication allows for many plugs in a number of parallel channels. The elements are magnetized by a homogenous external ac magnetic field. Reproducible experiments have been performed showing that the systems work and that the geometry of the elements, distance of the elements to the channel, and distance between the magnetic elements have a strong influence on the mixing efficiency and amount of magnetic beads in a plug. More work has to be done to understand the detailed influence of these parameters.

This work shows that it is possible to create many plugs of magnetic beads in a microchannel and that these must have efficient contact with the liquid since they induce mixing. This is therefore a promising method for performing biological separation using functionalized magnetic beads.

\footnotetext{
${ }^{1}$ A. Rida and M. A. M. Gijs, Anal. Chem. 76, 6239 (2004).

${ }^{2}$ K. Smistrup, T. Lund-Olesen, P. T. Tang, and M. F. Hansen, J. Appl. Phys. 99, 08P102 (2006).

${ }^{3}$ T. Lund-Olesen, H. Bruus, and M. F. Hansen, Biomed. Microdevices 9, 195 (2007).
} 